\title{
APPLICATION OF THE ARTEC EVA SCANNER FOR ORTHOTICS IN PRACTICE
}

\author{
Viktória Krajňáková, Viktória Rajtúková, Radovan Hudák, Jozef Živčák \\ Department of Biomedical Engineering and Measurement, Faculty of Mechanical Engineering, \\ Technical University of Košice, Košice, Slovakia
}

\begin{abstract}
This article deals with methodology and possibilities of using the Artec Eva scanner (Artec 3D, Luxembourg) in the field of prosthetics and orthotics with a focus on the part of face and neck. The methodology has been divided into 5 basic parts-environment preparation, scanner, subjects, scanning, and 3D modelling. The practical part includes 3D images of selected subjects representing a sample covering various types, especially in terms of the hair, beard, and eye area. Scanner outputs can be used in medicine for the manufacture of prostheses and dental implants and in the design of remodeling and other orthoses on the head.
\end{abstract}

Keywords

3D scanner, scanning, Artec Eva

\section{Introduction}

3D scanning is currently the most effective method for obtaining morphology of the surface of an object. Scanning results in a three dimensional imagea model. Appropriately chosen scanning technology is mainly reflected in the quality and accuracy of the 3D model, which is becoming ever more demanding.

This topic is highly up-to-date in prosthetics and orthotics, as it offers innovative approaches to the design and modification of therapeutic, compensatory, and prophylactic aids. The basis for modelling is in capturing the morphology of the site as closely as possible, so scanning different types of hair in terms of color and structure (curly, smooth hair), beard, tattoo, and ear, where we verified what things the scanner is able to digitize. After putting down individual methodologies of using the scanner, its outputs scanner outputs were evaluated to obtain information for its subsequent use in practice. The goal was to determine the possibilities of using the scanner to scan face areas in detail.

\section{Material and methods}

\author{
3D Scanner
}

3D scanners are widely used in practice. In prosthetics and orthotics, the goal is to obtain a scan of a certain part of the human body. Thanks to these scans, it is possible to create a shape-precise prosthesis or brace which is manufactured to the patient's size and adapted to their requirements. It is possible to respect individual morphology and, based on this, to change pressure distribution-use to relieve sensitive areas and to transfer the loads to places easy to handle the pressure. The result is a reduction in costs and time in modelling itself. Requirements on scanners used in prosthetics and orthotics are specific. The main emphasis is placed on scanners that are able to compensate for movement during the scanning itself. No high demands are placed on the accuracy of scanning, as is the case with technical applications [1]. 


\section{Artec Eva}

Artec Eva (Artec 3D, Luxembourg) is a hand-held 3D scanner that allows one to create a 3D mid-size model relatively quickly depending on the complexity of the surface (for more information see Table 1). The scanner has a wide range of uses in the technical field in the automotive, civil construction, and medical engineering industries. The ergonomic handle helps to scan larger objects, e.g. human body, which is important if the scans are for medical research. The scanner is affordable as to its price and is controlled by employing intuition.

Table 1: Artec Eva Scanner Specifications [2].

\begin{tabular}{|c|c|}
\hline Resolution: & $0.5 \mathrm{~mm}$ \\
\hline Accuracy: & $0.1 \mathrm{~mm}$ \\
\hline Distance accuracy: & $0.03 \%$ at $1000 \mathrm{~mm}$ \\
\hline Texture resolution: & $1.3 \mathrm{Mpx}$ \\
\hline Light source: & Flash \\
\hline Working distance: & $400-1000 \mathrm{~mm}$ \\
\hline Field of view (closer): & $214 \times 148 \mathrm{~mm}$ \\
\hline Field of view (farther): & $536 \times 371 \mathrm{~mm}$ \\
\hline Maximum snap rate: & Up to $16 \mathrm{fps}$ \\
\hline Exposure time: & $0.0002 \mathrm{~s}$ \\
\hline Scan speed: & 2 mil. points $/ \mathrm{s}$ \\
\hline Output formats: & $\begin{array}{l}\text { AOP, ASC, CSV, OBJ, PLY, } \\
\text { STL, WRL }\end{array}$ \\
\hline Dimensions: & $\begin{array}{l}262 \times 158 \times 63 \mathrm{~mm} \\
(\mathrm{~h} \times \mathrm{w} \times \mathrm{d})\end{array}$ \\
\hline Minimal requirements: & $\begin{array}{l}\text { Intel }{ }^{\circledR} \text { Core } 5^{\mathrm{TM}} \text {, NVIDIA } \\
\text { GeForce } 400 \text { or higher } \\
\text { (with a } 1 \mathrm{~GB} \text { memory or } \\
\text { higher: } 12 \mathrm{~Gb} \text { RAM, } \\
300 \mathrm{MB} \text { of free space at } \\
\text { the hard disc }\end{array}$ \\
\hline OS system support: & $\begin{array}{l}\text { Windows } 7,8 \text { or } 10 \text {, } \\
64 \text { bit }\end{array}$ \\
\hline Weight: & $1.2 \mathrm{~kg}$ \\
\hline Price: & $13.700 €$ \\
\hline
\end{tabular}

\section{Methodology of using the scanner in P\&O}

The methodology for using the scanner in prosthetics and orthotics can be divided into several points from the preparation of the environment to the evaluation of the data obtained.

The preparation and diagnostic of the premises, temperature and humidity control, temperature in the room and its equipment are monitored as part of the spatial preparation methodology for the scanning process. The room in which the person being scanned is situated should meet the basic requirement of ensuring a constant ambient temperature through air conditioning. The temperature range should be maintained from $18^{\circ} \mathrm{C}$ to $25^{\circ} \mathrm{C}$. At a lower temperature, the scanned person may shake and sweat at higher temperatures, which would greatly affect the scanning.

Another requirement is room darkening, the reason for this being in avoiding by the sun rays during scanning, which are undesirable in the process itself. Appropriate artificial lighting greatly enhances the quality of the 3D model. The recommended artificial lighting is within the range of 30 to 50 lux. The noise in the room may also affect the model itself. This value should be as high as $50 \mathrm{~dB}$ in order for the subject to be able to concentrate. To work with the Artec Eva scanner (Artec 3D, Luxembourg), a minimum distance of approximately 1 meter between the scanner and the person being scanned should be ensured. Based on this requirement, the proposed room should have dimensions of at least $2 \times 2 \mathrm{~m}$ or more.

Scanning itself is very simple, as the scanner does not need to be calibrated. With this type of scanner, it is not possible to choose the optimal optics for scanning or lens change. The scanner is ready for use when the green LED light turns on when the scanner is turned on. In the process of digitizing, you need to manipulate the scanner, so the tripod is not needed $[3,4]$.

Before each scanning, the subject needs to be informed of the scanning process and also of all the conditions under which the scanning will be taking place. Obtaining the information on the subject, such as their gender, weight, age, and various diseases that would have an undesirable effect on scanning represent an important part of this methodology. This information is needed for statistical processing.

It is important to remember that this is a safe and relatively fast scanning using the structured white light technology [5].

\section{Scanning methodology}

Correct positioning of the body of the person being scanned is a prerequisite for obtaining a high-quality 3D model. If it is necessary to obtain the patient's dimensional sizes, it is necessary to pass over the entire subject with the scanner by 360 degrees. When designing partial orthoses (such as face masks, dorsal or palm orthoses, etc.), scanning a person is sufficient only from a particular angle of view.

The Remote Meter shows the working area (Figure 1A), which ranges from 400 to $1000 \mathrm{~mm}$, with the scanner's recommended scanning distance of about 600 to $700 \mathrm{~mm}$. The object should be kept as close as possible to the center of the field of view. Fast scanning can cause the overlapping images to be too small, as a result of which the image does not automatically collate with the previous one, and the scanner system enters the waiting mode. This is called a missed tracking (Figure 1B) that can occur if the scanner is very close or too far away from the detected object, or if it cannot capture the surface. In this case, it is necessary to return to with the scanner to the area having been scanned $[5,6]$. 

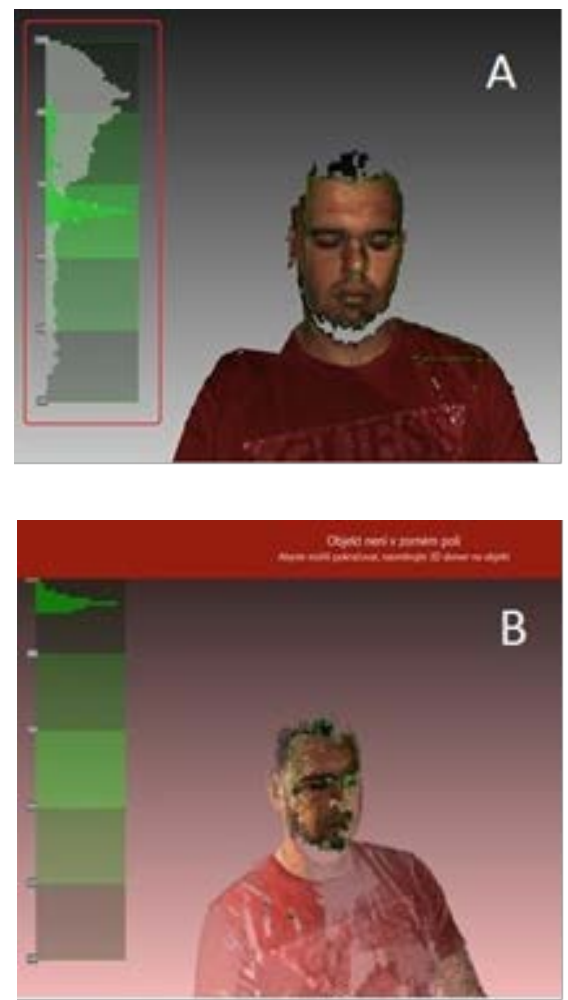

Fig. 1: A) Remote Meter with Recommended Subject Distance, B) Lost Tracking.

\section{Methodology of creating 3D models}

The autopilot is an important element in modelling. This is a complex mode that analyses the acquired data, resulting in the creation of the best possible output. The appropriate mode does not require any knowledge of algorithms, workflows, or individual settings [8, 13]. With Artec Studio software (Artec 3D, Luxembourg) creating SPROJ files, which are the project files for the software concerned, it is necessary to export the relevant models in the STL format (or another format suitable for possible further modifications), so that they can be opened on another device where no software from Artec is available. The format of the STL file, which is an abbreviation of the 3D printing technology called stereolithography, was developed by the company 3D Systems. This file describes three- dimensional surface geometry of the model and is most commonly used to export data to $3 \mathrm{D}$ printers from CAD software or other programmatic 3D modelers [7].

Export of scans takes a few minutes and depends mainly on the complexity of the object. They are mostly large files, because the human body is complex in shape and different structures are being changed in succession [8].

\section{Aspects of head and neck scanning}

Considerable attention has been paid to the hair scanning (Figure 2A), with the emphasis laid on

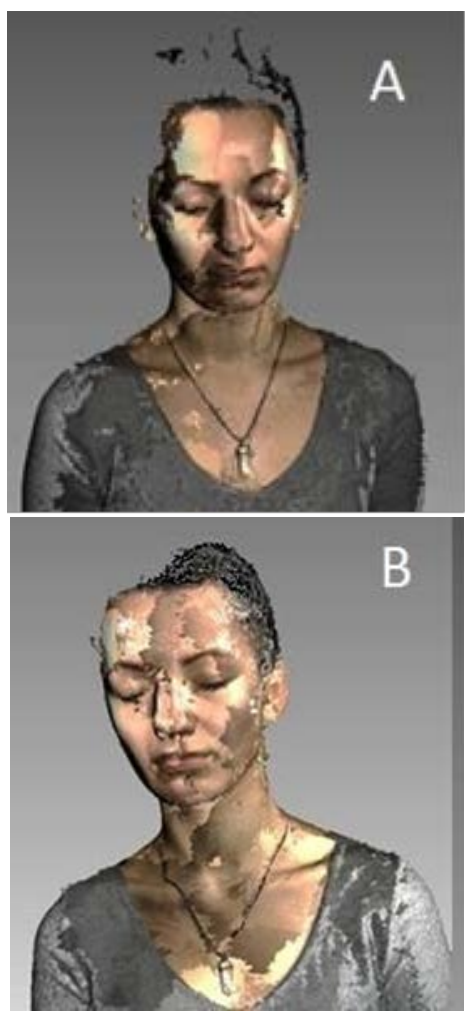

Fig. 2: Hair Scanning, A) Without water application, B) After water application.

different types of color (red, black, copper, brown, and blond hair) and texture (straight, curly hair).

In order to obtain a better 3D model, water applied to the hair was used to obtain a more detailed output, as demonstrated by the particular models (Figure 2B). As you can see after applying water to the hair, it was possible for the scanner to capture much of the hair compared to no water (Figure 2A). All the noted hair types are shown in Figure 3, where it is clear that hair color does not affect the scanning process.
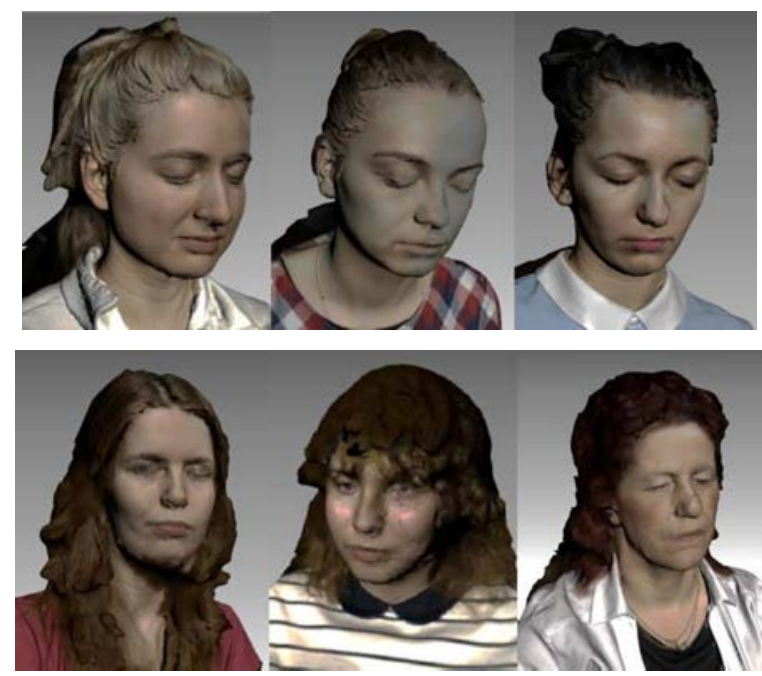

Fig. 3: Overview of the scanned hair types. 
The problem occurred when scanning the curled hair structure, where the scanner could not even capture their contour (Figure 4). The simplest and recommended scan is when the long hair is tied up $[9,10]$.

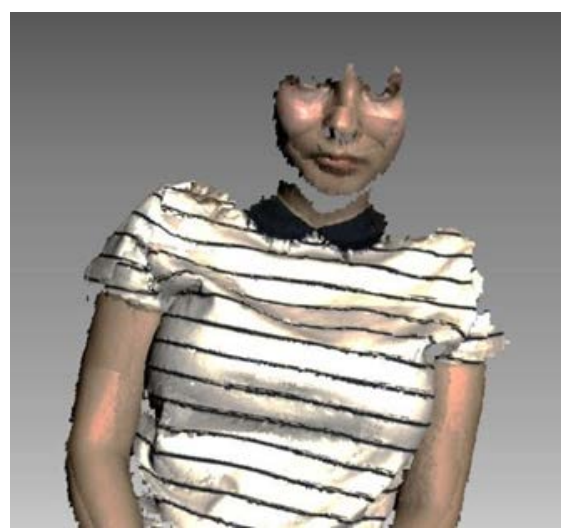

Fig. 4: Scanning curly hair.

The subject felt bad about scanning his eyes because the scanner has a high frequency of LED flashing. It is important to remind that scanning cannot damage the subject's eyesight. It was assumed that the eye's color texture will not be captured on the 3D output model of the person being captured because the cornea itself is clear and has a glossy surface. As can be seen in Figure 5, the scanner has completely scanned the eyes and their area adjacent area.

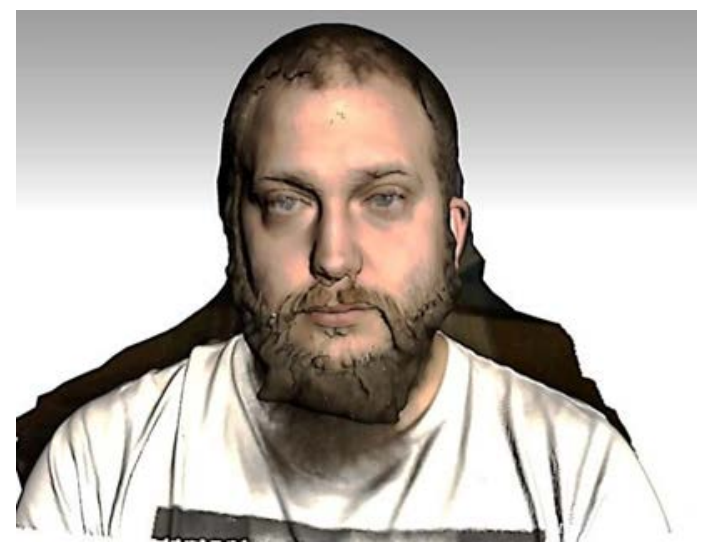

Fig. 5: Shooting eyes and thick beard.

Then followed scanning of the subject wearing the eyeglasses because it was assumed that the reflection from the glasses would make it impossible to scan the eye area, or the resulting model would not correspond to the real subject as to the form. The result of scanning a subject with glasses was surprising, as the scanning took place without undesirable phenomena. On the resulting 3D model, which is shown in Figure 6, it may be seen that the eyeglasses frame is not captured in detail.

During the beard scanning process, it was found out that the scanner can scan both a short bead as well as moustache. When scanning a thicker and longer beard, the scanner was unable to capture the beard hair ends.
As can be seen in Figure 5, the end of the subject's beard hair is blurred. This may be due to the fact that the ends have been greatly split.

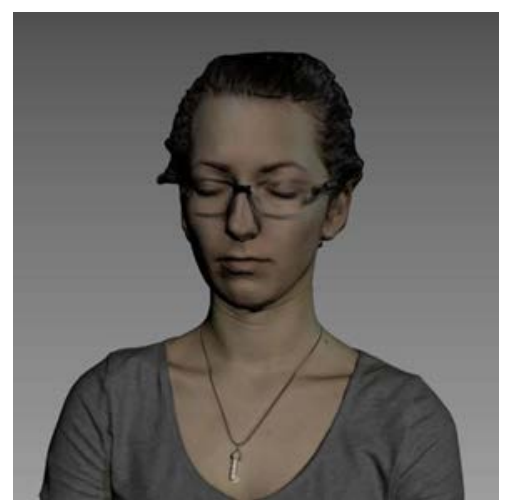

Fig. 6: Model with eyeglasses.

It was being determined by further scanning whether the scanner can capture complex shapes, such as an ear. The scanning of the ear can be performed in order to use the output from the scanner in the production of the epithesis of a natural appearance. It can be attached to the patient by a special adhesive or by magnetic implants. The ear, due to its smaller dimensions and complicated shape, was accurately scanned in great detail (Figure 7).

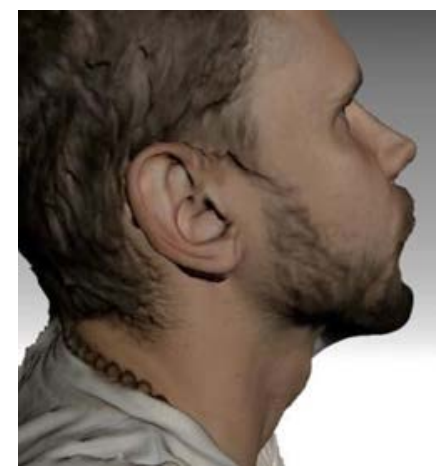

Fig. 7: Ear Model.

\section{Conclusion}

Quality scanning on the shoulder, neck and face is of great importance for the use in multiple areas. Scanner outputs can be used in medicine for the manufacture of prostheses and dental implants and in the design of remodeling and other orthoses on the heads of suckling infants, simulation before and after plastic surgery, preservation of cultural heritage, virtual reality, and so on $[11,12]$.

Based on the information gathered from the Artec Eva manual scanning process itself, it is possible to clearly confirm that this device is capable of capturing all the colored hair types. When scanning the curly hair structure, it was impossible to capture their contour. The 
autopilot of Artec Studio (Artec 3D, Luxembourg) was used to complete the capture of the subject, creating a 3D model and partially processed captured texture. The resulting model conforms to the template and matches the scanned subject. It is recommended to scan the subject with their hair tied up.

Hair scanning was also performed with water applied. During the scanning process itself, a difference was seen regarding hair digitization. Similarly, the resulting 3D model was more detailed compared to water-free comparison.

Complicated shapes, such as an ear or a complete tattoo pattern, could be seen without any complication. Short beard and moustache were not a problem factor for scanning. In the case of a thicker beard hair, the scanner was unable to capture the broken ends and the resulting model in the neck area is blurred.

\section{Acknowledgement}

This work was supported by the VEGA 1/0971/16Development and construction of low-cost modular proteins from the upper limbs produced by complementary technologies.

\section{References}

[1] Lovato C, Castellani U, Zancanaro C, \& Giachetti A. 2014. Automatic labelling of anatomical landmarks on 3D body scans. Graphical Models [internet]. 2014. 76(6), 648-657. DOI: 10.1016/j.gmod.2014.07.001

[2] Freedee. [internet] 2017 [cited 2017April 17]. Available from: http://www.freedee.hu/en/products/3d-scanners/artec-evaspecifications/

[3] Pandey G, Mcbride J, Savarese S, \& Eustice R. Extrinsic calibration of a 3D laser scanner and an omnidirectional camera. IFAC Proceedings Volumes [internet]. 2017 [cited 2016 April 21]. DOI: 10.3182/20100906-3-IT-2019.00059

[4] Anwar H. Calibrating projector flexibly for a real-time active 3D scanning system. Optik. [internet] 2018 [cited 2018 Jan 5]. 158,1088-1094. DOI: 10.1016/j.ijleo.2018.01.005

[5] Dúbravčík M. Prostriedky digitalizácie: Technická univerzita v Košiciach [internet] 2005 [cited 2018 April 21]. Availiable from:

http://www.sjf.tuke.sk/transferinovacii/pages/archiv/transfer/82005/pdf/52-54.pdf
[6] Herbort S, Gerken B, Schugk D, Wöhler C. 2013. 3D range scan enhancement using image-based methods. ISPRS Journal of Photogrammetry and Remote Sensing, [internet] 2017 [cited 2017 Aug. 7]. 84, 69-84. DOI: 10.1016/j.isprsjprs.2013.07.004

[7] Čelko J, Kováč M, Kotek P. Analysis of the Pavement Surface Texture by 3D Scanner. Transportation Research Procedia, 14, [internet] 2016. [cited 2017 Aug. 7]. 2994-3003. DOI: $10.1016 / j . t r p r o .2016 .05 .434$

[8] Rusinkiewicz S, Hall-Holt O, Levoy M. Real-time 3D model acquisition. ACM Transactions on Graphics, 21, [internet] 2002. [cited 2017 April 7] 438-446. DOI: 10.1145/566654.566600

[9] Modabber A, Peters F, Kniha K, Goloborodko E, Ghassemi A, Lethaus B, Möhlhenrich SC. Evaluation of the accuracy of a mobile and a stationary system for three-dimensional facial scanning. Journal of Cranio-Maxillofacial Surgery, [internet] 2016. [cited 2017 April 7] 44(10), 1719-1724.

DOI: $\underline{10.1016 / j . j c m s .2016 .08 .008}$

[10] Schweitzer W, Röhrich E, Schaepman M, Thali MJ, Ebert L. Aspects of 3D surface scanner performance for post-mortem skin documentation in forensic medicine using rigid benchmark objects. Journal of Forensic. 2013.

[11] Shinomiya A, Shindo A, Kawanishi M, Miyake K, Nakamura T, Matsubara S, Tamiya T. Usefulness of the 3D virtual visualization surgical planning simulation and $3 \mathrm{D}$ model for endoscopic endonasal transsphenoidal surgery of pituitary adenoma: Technical report and review of literature. Interdisciplinary Neurosurgery: Advanced Techniques and Case Management, [internet] 2018. [cited 2017 November 13], 13-19. DOI: $10.1016 / j$.inat.2018.02.002

[12] Wibirama S, Nugroho HA, Hamamoto K. 2017. Evaluating 3D gaze tracking in virtual space: A computer graphics approach. Entertainment Computing, [internet] 2018. [cited 2017 November 13] 21, 11-17. DOI: $10.1016 /$ j.entcom.2017.04.003

[13] Lee W, Lee B, Yang X, Jung H, Bok I, Kim C, You H. 2018. A 3D anthropometric sizing analysis system based on North American CAESAR 3D scan data for design of head wearable products. Computers and Industrial Engineering 117, [internet] [cited May 2017] 121-130. DOI: 10.1016/j.cie.2018.01.023

Ing. Viktória Krajňáková Department of Biomedical Engineering and Measurement

Faculty of Mechanical Engineering Technical University of Košice Letná 9, 04200 Košice

E-mail: viktoria.krajnakova@tuke.sk Phone: +421556022043 